\title{
Edge Preserving Bit-Plane Adaptive Wiener Filter for Gaussian Noise Restoration
}

\author{
Rashi Agarwal \\ Information Technology Department at University Institute of Engineering and Technology, \\ Chhatrapati Shahu Ji Maharaj University, Kanpur, Uttar Pradesh, India.
}

\begin{abstract}
Restoration of images is a significant area of research as presence of noise is unavoidable. Noises are modeled as Gaussian noise predominantly in all fields of image processing on images acquired from all spectrums. The global method of Adaptive Wiener Filter seems to perform the best amongst all the filters available. Numerous studies have established this fact. We have tried to develop a local bitplane Adaptive Wiener Filter Algorithm which performs much better than the traditional filter. The results are not only visually verifiable but the Root Mean Square Error and the Peak Signal to Noise Ratio parameters validate our results. The suggested algorithm is actually a modification of the original one hence the processing time is not significantly effected. The algorithm works because instead of the global method which blurs out the edges, the local bitplane method works on the windows in the bitplane and hence the edges are preserved in a much better way. The results are more dominant in cases with larger variance noise and larger window size which tends to get blurred more in the global method.
\end{abstract}

\section{Keywords}

Image Restoration, Wiener Filter, Adaptive Wiener Filter, Gaussian Noise, Bitplanes

\section{INTRODUCTION}

Noise is present in all images, be it satellite images or medical images or astronomical images or images acquired from our cameras. The noise is introduced due to various reasons, such as during image formation process, the recording or transmission etc. All applications on digital images focus on processing of the images to either get a better image as per the suitability of the application or by extraction of features to perform identification or classification. An image contains a variety of information such as edge and plane regions, thus a powerful noise reduction with keeping the original image signal is desired. All applications focus on parameters related to the image intensity which gets distorted on even a slight presence of noise.

In this paper, we consider restoration of an image corrupted by additive white Gaussian noise. The Adaptive Wiener Filter (AWF) is the most popular denoising method for additive white Gaussian noise [1]. The AWF is an adaptation of the popular Wiener filter which uses the central pixel value and local statistical value, hence the filtering process is adaptive, which results in a high quality restored image. There are problems with this as, if the degraded image is of low Signalto-Noise Ratio (SNR), some noises remain in the image processed by the AWF, because the estimated noise value is far from the correct one. On the other hand, if the degraded image is of high SNR, the edge components become too smooth in the image processed by the AWF. This means that the original image information is distorted. In this paper we first study the distribution of noise over the bitplanes of the image and based on the results we use the AWF on each of the bitplanes to get a resultant image which produces better image quality than the result got from application of AWF on the corrupted image.

\section{METHODOLOGY}

\subsection{Wiener Filter}

There are three standard noise models[2], which appropriately portray the types of noises encountered in most images. These are additive noise, multiplicative noise and impulse noise. According to the central limit theorem, when several random numbers are added, the sum tends to be Gaussianly Distributed. As mentioned before, there are various sources of noise in an image, such as instrument nose, quantization noise, transmission noise etc. We thus, assume that all these noise components combined may be modeled as Gaussian noise. Gaussian noise is the type of noise in which, at each pixel position $(i, j)$ the random noise value, that affects the true pixel value, is drawn from a Gaussian probability density function with mean mu(i,j) and standard deviation sigma $(\mathrm{i}, \mathrm{j})$. This noise affects all pixel values. Image noise is often assumed to be additive, zero-mean. unbiased, independent, uncorrelated, homogeneous, white, Gaussian and identically distributed.

The use of Wiener filter has been well established to remove Gaussian noise[3]. If $\widehat{\mathrm{f}(\mathrm{r})}$ is an estimate of the original undegraded image $f(r)$, we wish to calculate $\widehat{f(r)}$ so that the norm of the residual image $f(r)-\widehat{f(r)}$ is minimal over all possible versions of image $f(r)$. This is equivalent to saying that we wish to identify $\widehat{\mathrm{f}(\mathrm{r})}$ which minimizes:

$$
\mathrm{e}^{2} \equiv \mathrm{E}\left\{|\mathrm{f}(\mathrm{r})-\widehat{\mathrm{f}(\mathrm{r})}|^{2}\right\}
$$

To minimize the mean square error, the value of the filter obtained was given by the expression:

$$
\widehat{M}(u, v)=\frac{H^{*}(u, v)}{|\widehat{H(u, v)}|^{2}+\frac{S_{v v}(u, v)}{S_{f f}(u, v)}}
$$

where $H^{*}(u, v)$ is the Fourier transform of the point spread function of the degradation process $H(u, v)$ its complex conjugate, $S_{v v}(u, v)$ is the spectral density of the noise field and $S_{f f}(u, v)$ is the spectral density of the undegraded image. $\widehat{M}(u, v)$ is known as the Wiener filter for image restoration.

\subsection{Adaptive Wiener Filter}

A degraded image y can be represented as:

$$
\mathrm{y}=\mathrm{x}+\mathrm{n}
$$

where $\mathrm{x}$ is the original image and $\mathrm{n}$ is a zero mean additive white Gaussian noise whose standard deviation is $\sigma_{n}$.

The AWF is expressed by the following equations: 


$$
\begin{aligned}
& \hat{x}(i, j)=\frac{\sigma_{x}^{2}(i, j) \cdot y(i, j)+\sigma_{n}^{2} \cdot \bar{y}(i, j)}{\sigma_{x}^{2}(i, j)+\sigma_{n}^{2}} \\
& \sigma_{x}^{2}(i, j)=\max \left(0,+\sigma_{y}^{2}(i, j)-\sigma_{n}^{2}\right.
\end{aligned}
$$

where $\hat{\mathrm{x}}(\mathrm{i}, \mathrm{j})$ is the filtered output at $(\mathrm{i}, \mathrm{j}),), \sigma_{n}{ }^{2}$ is the noise variance which is constant over the image, and $y(i, j)$ and $\sigma_{y}^{2}(i, j)$ are the local mean and local variance of the input image $y$ at $(i, j)$. [4]

The output is calculated using the central pixel value and local statistics, it means that the filtering process is not constant for each pixel. The AWF provides a good performance for the degraded image corrupted by additive white Gaussian noise. However, if the SNR of the degraded image is low, remaining noises appear in the restored image. Hence, the performance quality by the AWF is limited. AWF changes its behavior based on the statistical characteristics of the image inside the filter window. Adaptive filter performance is usually superior to non-adaptive counterparts. But the improved performance is at the cost of added filtercomplexity. Mean and variance are two important statistical measures using which adaptive filters can be designed.

\subsection{Bit-plane Adaptive Wiener Filter}

Given an X-bit per pixel image, slicing the image at different planes (bit-planes) plays an important role in image processing. In general, 8-bit per pixel images are processed. We can slice an image into the following bitplanes. Zero is the least significant bit (LSB) and 7 is the most significant bit (MSB) [5]. The performance evaluation of the filtering operation is quantified by the PSNR (Peak Signal to Noise Ratio) and MSE (Mean Square Error) calculated using formula:

$$
P S N R=10 \log _{10}\left(\frac{255^{2}}{M S E}\right)
$$

where

$$
M S E=\frac{1}{M N} \sum_{i=1}^{M} \sum_{i=1}^{N}[g(i, j)-f(i, j)]^{2}
$$

Here $\mathrm{M}$ and $\mathrm{N}$ are the total number of pixels in the horizontal and the vertical dimensions of image, $g$ denotes the Noise image and $f$ denotes the filtered image.

To understand how the additive Gaussian noise effects the bitplanes of an image, the following steps were taken:

i. We observed an 8 bit grayscale image and added noises with zero mean and various variance ranging from 0.01 to 0.2 in intervals of 0.02 .

ii. The bitplanes of the corrupted image were extracted.

iii. We applied AWF on each bitplane.

iv. Next we calculated the PSNR between the corresponding bitplanes of the corrupted image and the AWF applied bitplanes.

v. The AWF was applied on the corrupted image.

vi. The bitplanes were extracted for this restored image too.

vii. The PSNR between the corresponding bitplanes of the AWF restored image and the corrupted bitplanes was calculated.

viii. Plots were made to see the results.

The following pattern was observed:

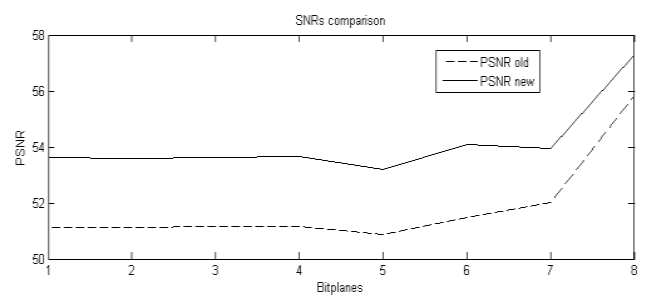

Fig 1: Comparison of RMSE between bitplanes

Figure1 clearly showed that the AWF applied bitplanes give a better PSNR than the one in which AWF was applied on the entire image. This is because AWF works on the local parameters of the window around it and hence AWF performs better on the local bitplanes. Due to this promising result AWF was applied on all bitplanes and then the corresponding bitplanes were added as per their order to get back the combined image.

\section{RESULTS AND DISCUSSIONS}

The bitplanes extracted were restored with the AWF as shown the following figures.

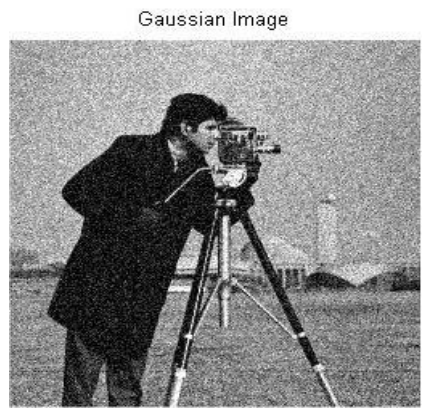

Fig 2: Image corrupted with variance $=0.05$

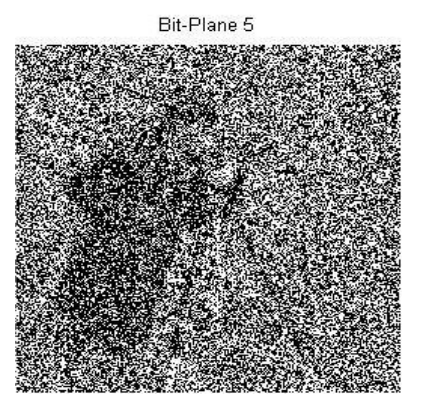

Fig 4: Original corrupted bitplane 5

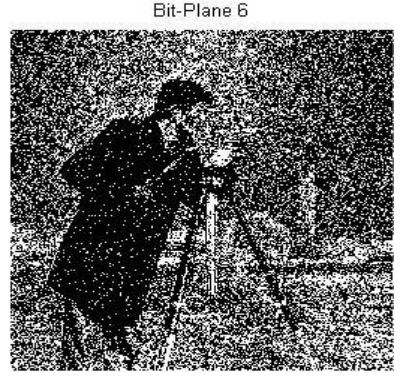

Fig 6 : Original corrupted bitplane 6

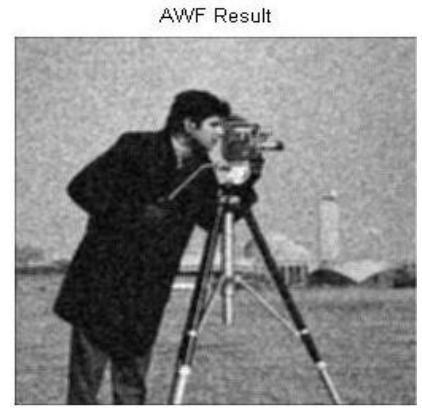

Fig 3: AWF filtered image with window $\operatorname{size}=7$

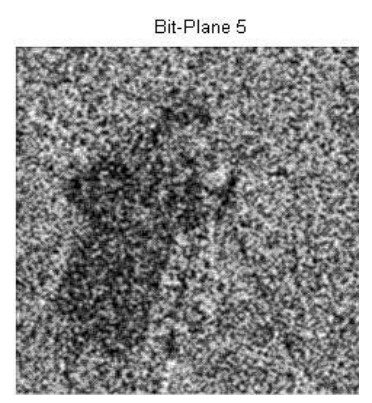

Fig 5 : AWF bitplane 5

Bit-Plane 6

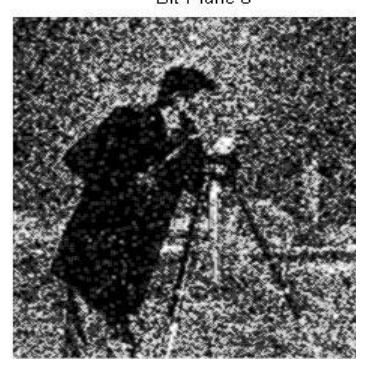

Fig 7: AWF bitplane 6 


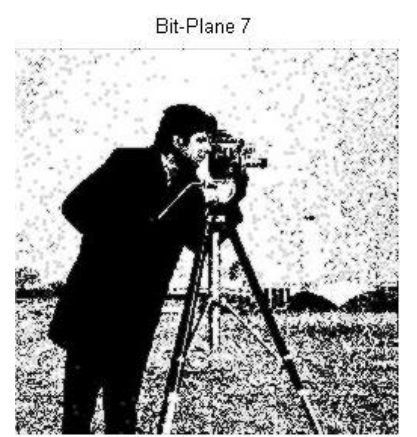

Fig 8 : Original corrupted bitplane 5

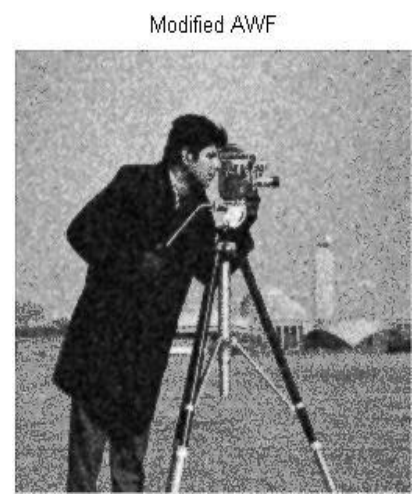

Fig 10: Combined image from all modified AWF applied bitplanes

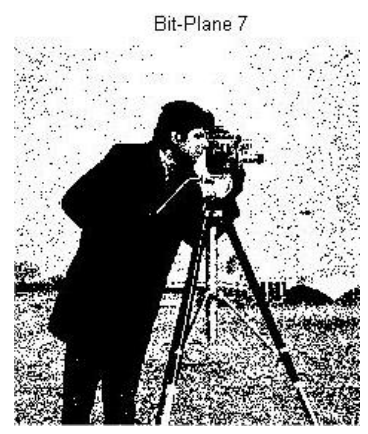

Fig 9 : AWF bitplane7

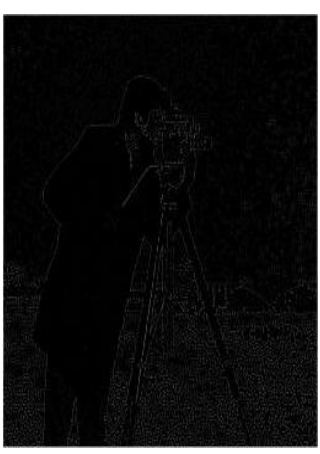

Fig 11: Difference between modified AWF and AWF images

The bitplanes 0 to 4 did not show any significant change which is apparent in Fig. 1 too. But the bitplanes 5 to 7 gave a better image and hence the combined values gave a mich better result. Figure 11 clearly shows that the difference between the two algorithms preserves the edges better. The values RMSE andPSNR were extracted for different setof 10 images, such as 'Lena', 'Cameraman', 'Peppers' etc. and all were corrupted with noises ranging from variance. deviation of 0.01 to 0.2 in an interval of 0.02 . All corrupted images were restored with both AWF algorithm and the modified bitplane AWF algorithm with the window sizes of $3 * 3,5 * 5$, $7 * 7,9 * 9,11 * 11,13 * 13,15 * 15$ and $17 * 17$. The following plots were observed:

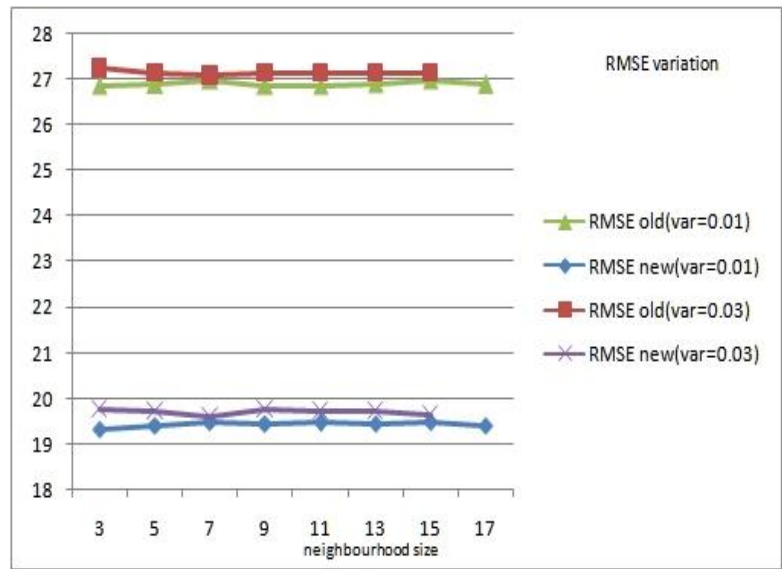

Fig 12 : RMSE for the variances $=0.01$ and 0.03 against AWF applied images and modified AWF images(with various window sizes)

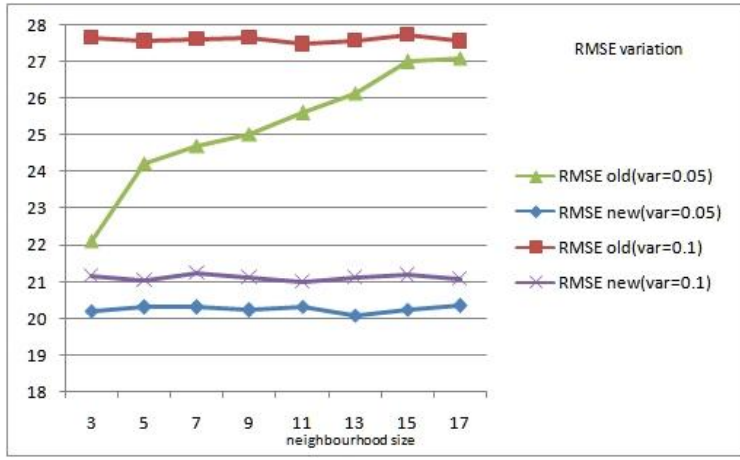

Fig 13: RMSE for the variances $=0.05$ and 0.1 against AWF applied images and modified AWF images (with various window sizes)

It is clearly observed that the RMSE decreases significantly for the modified AWF algorithm. This is more pronounced in values with larger window size as the global algorithm which works on the entire image tends to blur the images and we loose the edges. The local operator performs better and preserves the edges

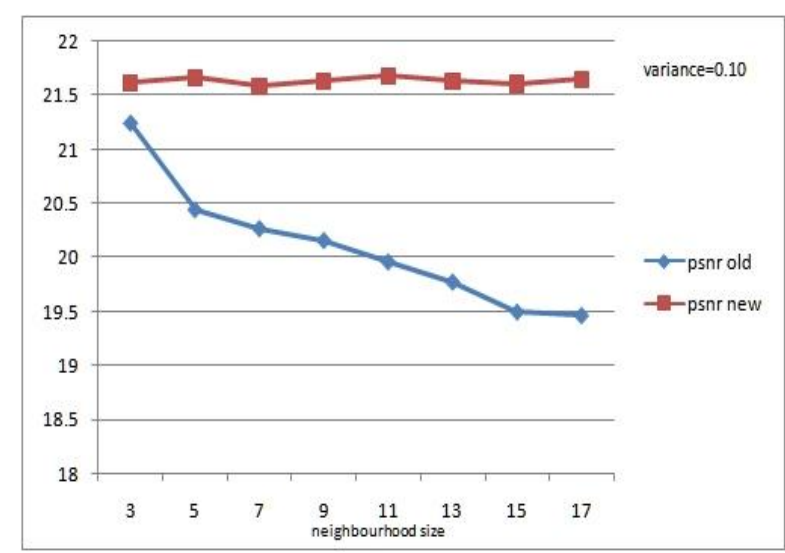

Fig 14 : PSNR for the variance $=0.1$ against $A W F$ applied images and modified AWF images( with various window sizes)

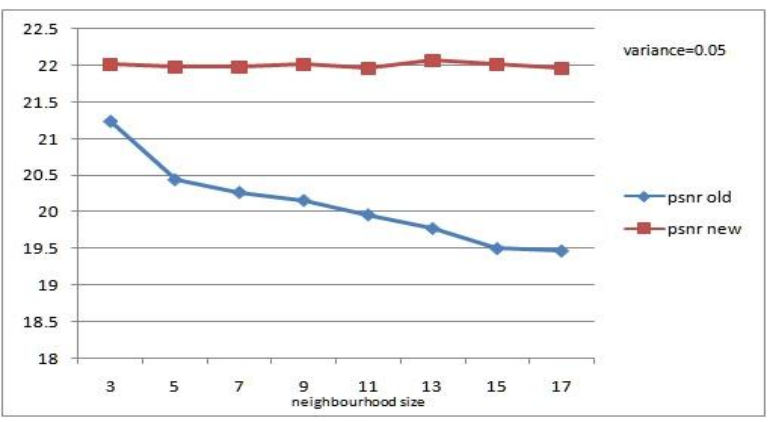

Fig 15 : PSNR for the variance $=0.05$ against $\mathrm{AWF}$ applied images and modified AWF images( with various window sizes)

The PSNR shows significant reduction specially in the bigger window size values.

\section{CONCLUSION}

The visual results of the images, the values of the RMSE and PSNR values conclude that our modified bitplane AWF algorithm perform better than the global AWF algorithm. The processing time showed no significant difference as the 
principal of the algorithm has notchanged. We are applying the algorithm on the local level rather than the global level. The algorithm works because instead of the global method which blurs out the edges, the local bitplane method works on the windows in the bitplane and hence the edges are preserved in a much better way. The results are more dominant in cases with larger variance noise and larger window size which tends to get blurred more in the global method. The further scope of work would be to actually study the mathematical model of the work and derive the algorithms results on binary images since the bitplanes are actually binary images.

\section{REFERENCES}

[1] J. S. Lim, "Two-Dimensional Signal and Image Processing," Prentice-Hall, 1990.
[2] H. C. Andrews and B. R. Hunt, "Digital Image Restoration," Prentice-Hall, 1977.

[3] J. Giannoula, A.Classification-based adaptive filtering for multiframe blind image restoration. IEEE Trans. Image Proc., 20: 382-390,2011.

[4] Lopez-Martinez, J.L. and V. Kober., Blind Adaptive method for image restoration using microscanning. IEICE Trans. Inform. Syst., 950: 280-284, 2011.

[5] R. Agarwal, "Bit Planes Histogram Equalization for Tone Mapping of High Contrast Images", IEEE proceedings of Computer Graphics, Imaging and Visualisation Conference, Singapore, 33-38, 2011. 